\title{
Interaction Mechanisms of Giant Unilamellar Vesicles with Hydrophobic Glass Surfaces and Silicone Oil-Water Interfaces: Adsorption, Deformation, Rupture, Dynamic Shape Changes, Internal Vesicle Formation, and Desorption
}

\author{
Chiho Kataoka-Hamai* and Kohsaku Kawakami \\ International Center for Materials Nanoarchitectonics (WPI-MANA), \\ National Institute for Materials Science, 1-1 Namiki, Tsukuba, Ibaraki 305-0044, Japan \\ *E-mail: kataoka.chiho@nims.go.jp
}

Number of pages: 5

Number of figures: 2

\section{SUPPORTING INFORMATION -}

\section{Comparison of the Glass Surfaces Modified with ODTMS in the Vapor and Liquid Phases}

\section{METHODS}

Glass Surface Modification in a Liquid Phase. To examine the effect of the silanization procedures on vesicle adsorption, the glass surface was modified with octadecyltrimethoxysilane (ODTMS) in a liquid phase. ${ }^{1}$ The glass coverslips were cleaned via sonication in acetone and ethanol for $5 \mathrm{~min}$, which was conducted three times. After drying under a nitrogen stream, the coverslips were exposed to air plasma generated by a PDC-32G plasma cleaner (Harrick Plasma, Ithaca, NY, US) for $2 \mathrm{~min}$. The cleaned coverslips were immersed for $20 \mathrm{~h}$ in dodecane containing $4 \mathrm{mM}$ of ODTMS. After silanization, the coverslips were sonicated in chloroform for $5 \mathrm{~min}$, which was conducted three times. The water contact angle of the ODTMS-treated surface was $96.4^{\circ}\left( \pm 5.5^{\circ}, N=6\right)$. The treated coverslips were stored in ethanol until use.

Atomic Force Microscopy. Atomic force microscopy (AFM) imaging was performed using an MFP3D system (Asylum Research, Santa Barbara, CA, US). The ODTMS-modified glass was observed in air in AC mode with a scan rate of $4 \mu \mathrm{m} / \mathrm{s}$ using OMCL-AC160TS-C2 cantilevers $(\sim 42 \mathrm{~N} / \mathrm{m}, \sim 300 \mathrm{kHz}$, Olympus, Tokyo, Japan). 


\section{RESULTS}

AFM Observation of the ODTMS-Modified Glass Surfaces. To examine surface homogeneity, the ODTMS-modified glass surfaces were imaged using AFM (Figure S1). Holes with a depth of approximately $2 \mathrm{~nm}$ were observed (arrows) in both samples treated with ODTMS vapor (Figure S1A) and liquid ODTMS (Figure S1B). The hole depth is in good agreement with the thickness of the octadecylsilyl monolayers, ${ }^{2}$ indicating the presence of monolayer defects. The surface modified with ODTMS vapor was smoother than that modified in the solution. The root-mean-square surface roughness obtained after the vapor- and liquid-phase depositions was $183( \pm 7)$ and $311( \pm 6)$ pm, respectively $(N=$ 4-5).
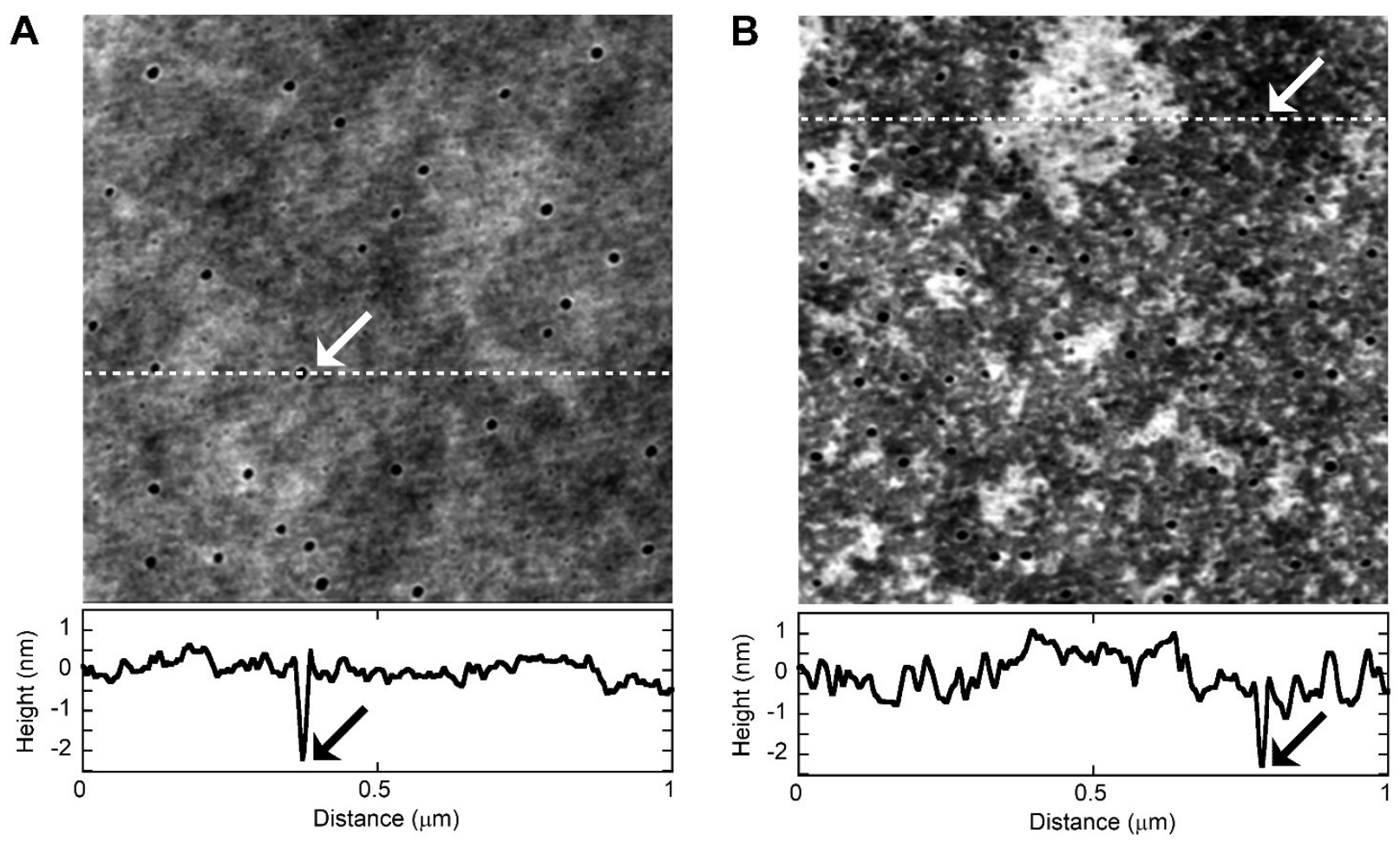

Figure S1. AFM data obtained for the glass surfaces modified with ODTMS in the vapor (A) and liquid (B) phases. Arrows indicate defects. Cross sections (shown in the lower panels) were measured along the dashed lines in the images.

Vesicle Adsorption on the ODTMS-Modified Glass. In order to examine the effect of the silanization procedures on lipid adsorption, fluorescence imaging was performed after adsorption of DOPC/DOPS/TR-DHPE (79.2:19.8:1) giant unilamellar vesicles (GUVs) (Figure S2). The feasibility of the area expansion of the lipid monolayer patches was strongly dependent on the surface modification 
protocols. On the surface treated in the vapor phase (Figure S2A), no patch structures were observed. The results are consistent with the time-resolved data recorded for the GUV rupture events (Figure 3), where lipid migration occurred immediately after vesicle rupture. In contrast, lipid monolayer patches were clearly visualized on the surface modified in the liquid phase (Figure S2B). It therefore seems that the lipids did not freely migrate on the liquid-phase-treated surface.

To further examine the effect of the ODTMS film quality, planar lipid monolayers were formed by adsorption of DOPC/DOPS/TR-DHPE (79.2:19.8:1) large unilamellar vesicles (0.7 mM lipid, 100-nm diameter) for $5 \mathrm{~h}$. After washing with buffer to remove unbound vesicles, the supported monolayers were characterized by fluorescence recovery after photobleaching experiments. The planar monolayers on the surface modified in the solution were mobile $\left(F=0.95( \pm 0.0060), D=1.5( \pm 0.11) \mu \mathrm{m}^{2} / \mathrm{s}, N=4\right)$. However, the diffusion constant was four times smaller than that determined for the monolayers supported on the glass surface modified with ODTMS vapor $\left(D=6.0( \pm 0.79) \mu \mathrm{m}^{2} / \mathrm{s}\right.$, see text for details $)$. The results revealed that the lipid migration was hampered on the hydrocarbon films attached in the liquid phase.
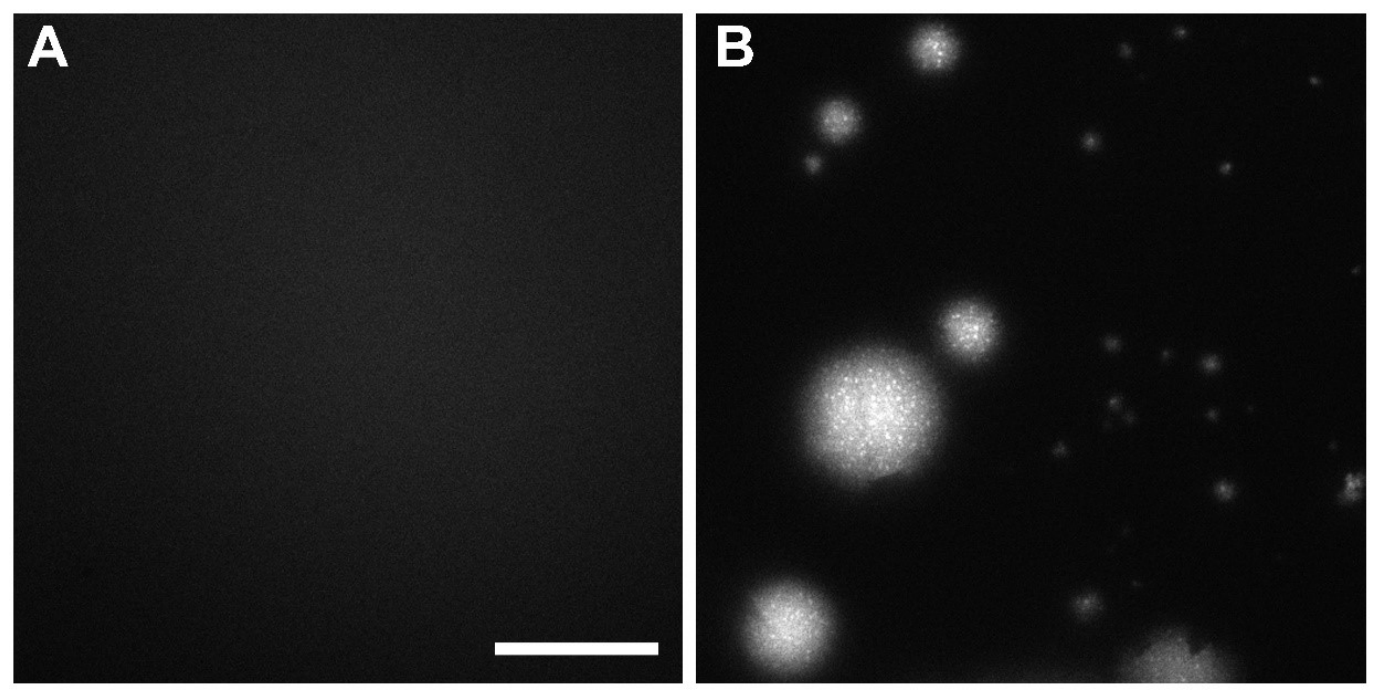

Figure S2. Fluorescence images obtained after adsorption of DOPC/DOPS/TR-DHPE (79.2:19.8:1) GUVs on the glass surfaces modified with ODTMS in the vapor (A) and liquid (B) phases. The surfaces were incubated for $30 \mathrm{~min}$ with GUVs $(0.2 \mu \mathrm{M}$ lipid) and then washed with buffer for the fluorescence observation. The data were recorded with $40 \times$ magnification. Scale bar: $50 \mu \mathrm{m}$; both images use the same scale. 


\section{Movie Legends}

\section{Movie 1}

Movie of the multilamellar vesicle rupture event on ODTMS-modified glass shown in Figure 1D. The image size is $14.4 \times 14.4 \mu^{2}$. Each frame was acquired in $26 \mathrm{~ms}$, and play back speed is 10 frames per second ( 92 frames total).

\section{Movie 2}

Movie of the multilamellar vesicle rupture event on the silicone oil-water interface shown in Figure 1E. The image size is $21.4 \times 21.4 \mu \mathrm{m}^{2}$. Each frame was acquired in $39 \mathrm{~ms}$, and play back speed is 10 frames per second (43 frames total).

\section{Movie 3}

Movie of the spontaneous GUV rupture event on HMDS-modified glass shown in Figure 2B. The image size is $9.6 \times 9.6 \mu \mathrm{m}^{2}$. Each frame was acquired in $3 \mathrm{~ms}$, and play back speed is 10 frames per second (66 frames total).

\section{Movie 4}

Movie of the spontaneous GUV rupture event on ODTMS-modified glass shown in Figure 3B. The image size is $20.4 \times 20.4 \mu \mathrm{m}^{2}$. Each frame was acquired in $3 \mathrm{~ms}$, and play back speed is 10 frames per second (100 frames total).

\section{Movie 5}

Movie of the spontaneous GUV rupture event on the silicone oil-water interface shown in Figure 5B. The image size is $9.6 \times 9.6 \mu \mathrm{m}^{2}$. Each frame was acquired in $3 \mathrm{~ms}$, and play back speed is 10 frames per second (19 frames total).

\section{Movie 6}

Movie of the dynamic deformation event on the silicone oil-water interface shown in Figure 6D (0-1.002 s). The image size is $6.4 \times 6.4 \mu \mathrm{m}^{2}$. Each frame was acquired in $59 \mathrm{~ms}$, and play back speed is 10 frames per second (50 frames total).

\section{Movie 7}

Movie of the adsorbed GUV with an internal vesicle on the silicone oil-water interface shown in Figure $6 \mathrm{D}$ ( $3 \mathrm{~min} 26.044-28.077 \mathrm{~s}$ ). The image size is $6.4 \times 6.4 \mu \mathrm{m}^{2}$. Each frame was acquired in $59 \mathrm{~ms}$, and play back speed is 10 frames per second ( 50 frames total). 


\section{REFERENCES}

1. Zan, G. H.; Tan, C. M.; Deserno, M.; Lanni, F.; Lösche, M., Hemifusion of Giant Unilamellar Vesicles with Planar Hydrophobic Surfaces: A Fluorescence Microscopy Study. Soft Matter 2012, 8, 10877-10886.

2. Mezger, M.; Reichert, H.; Schöder, S.; Okasinski, J.; Schröder, H.; Dosch, H.; Palms, D.; Ralston, J.; Honkimäki, V., High-Resolution in Situ X-Ray Study of the Hydrophobic Gap at the Water-OctadecylTrichlorosilane Interface. Proc. Natl. Acad. Sci. U.S.A. 2006, 103, 18401-18404. 